\title{
Erratum: Dynamics of the inner crust of neutron stars: Hydrodynamics, elasticity, and collective modes [Phys. Rev. C 87, 055803 (2013)]
}

\author{
D. Kobyakov and C. J. Pethick
}

(Received 17 October 2016; published 23 November 2016)

DOI: 10.1103/PhysRevC.94.059902

Due to a coding error, the numerical results for the thermodynamic derivatives and mode velocities are incorrect. The corrected results may be found in Ref. [1].

We also take this opportunity to correct some typos.

The last term in the sum in Eq. (31) should read $E_{n n} n_{n}^{k} n_{n}^{l}$.

Equation (38) should be

$$
v_{n p}^{4}-v_{n}^{2} v_{p}^{2}=-n_{p}^{2}\left(n_{n}^{s}\right)^{2}\left(E_{n n}\left[E_{p p}+4 S / 3 n_{p}^{2}\right]-E_{n p}^{2}\right) /\left(\rho^{s} \rho^{n}\right) .
$$

Equation (44) should be

$$
\partial \tilde{\mu} / \partial Y_{e}=\partial \hat{\mu} / \partial Y_{e}-\partial \mu_{e} / \partial Y_{e}=(1 / n) \partial^{2} E / \partial Y_{e}^{2} .
$$

[1] D. Kobyakov and C. J. Pethick, Phys. Rev. C 94, 055806 (2016). 